OPEN ACCESS

Edited by:

Holger Wille,

University of Alberta, Canada

Reviewed by:

Alina Gonzalez-Quevedo, Instituto de Neurología y Neurocirugía,

La Habana, Cuba

Peng Lei,

Sichuan University, China

*Correspondence:

Liyong Wu

wmywly@hotmail.com

Specialty section:

This article was submitted to

Dementia and Neurodegenerative

Diseases,

a section of the journal

Frontiers in Neurology

Received: 19 December 2021

Accepted: 21 January 2022

Published: 14 February 2022

Citation:

Kong Y, Chen Z, Zhang J and Wu L

(2022) Erythrocyte Indices in Creutzfeldt-Jakob Disease Predict

Survival Time.

Front. Neurol. 13:839081. doi: 10.3389/fneur.2022.839081

\section{Erythrocyte Indices in Creutzfeldt-Jakob Disease Predict Survival Time}

\author{
Yu Kong, Zhongyun Chen, Jing Zhang and Liyong $W u^{*}$ \\ Department of Neurology, Xuanwu Hospital, Capital Medical University, Beijing, China
}

Background: Creutzfeldt-Jakob disease (CJD) is a devastating neurodegenerative disease caused by propagation of abnormally folded prion proteins $\left(\mathrm{PrPc}^{\mathrm{Sc}}\right)$. Some fluid biomarkers have been reported to be associated with disease duration in CJD. Based on studies which have found that prion protein $\left(\mathrm{PrP}^{\mathrm{C}}\right)$ played a role in erythrocytic hematopoiesis, we evaluated the association between peripheral red blood cell indices and survival time in CJD.

Methods: We retrospectively collected data on peripheral red blood cell indices, including red blood cell (RBC) count, hemoglobin $(\mathrm{Hb})$, hematocrit $(\mathrm{HCT})$, mean corpuscular volume (MCV), mean corpuscular hemoglobin $(\mathrm{MCH})$, mean corpuscular hemoglobin concentration (MCHC), and red cell distribution width (RDW), from 125 CJD patients. Cox proportional hazard models were generated to determine whether red cell indices correlated with survival time of patients with CJD.

Results: Of the 125 included participants, 70 (56\%) were male, and the mean age at diagnosis (SD) was 60.3 (9.5) years. Hemoglobin levels (hazard ratio 1.710, 95\% Cl $1.124-2.600, p=0.012$ ) and HCT (hazard ratio 1.689, 95\% Cl 1.112-2.565, $p=0.014$ ) were significantly associated with survival time after controlling for sex, age, and Barthel Index. Red blood cell count, MCV, MCH, MCHC, and RDW were not associated with survival time before or after adjusting for covariates.

Conclusions: Our study found that $\mathrm{Hb}$ and HCT were significantly associated with survival time in patients with CJD. These results may inform evaluation of the mechanisms of interaction between prion disease and hematopoiesis, and indicate that $\mathrm{Hb}$ and HCT may be potential prognostic biomarkers.

Keywords: Creutzfeldt-Jakob disease, survival time, hemoglobin, hematocrit, erythrocyte indices, red cell indices

\section{INTRODUCTION}

Creutzfeldt-Jakob disease (CJD) is a rapidly progressive and fatal neurodegenerative disease caused by the propagation of abnormally folded prion proteins $\left(\mathrm{PrP}^{\mathrm{Sc}}\right)(1)$. The survival time of patients with CJD is variable, and ranges from weeks to several years, with a typical duration of 4 to 6 months $(2-5)$. The speed at which CJD progresses and the fact that it is incurable highlights the importance of prediction of disease duration for disease management and clinical trial design. Several studies have found that several fluid biomarkers, such as cerebrospinal fluid (CSF) and plasma total tau (t-tau) levels, neurofilament light (NFL), the ratio of $\mathrm{t}$-tau to phosphorylated tau (p-tau), and the 
14-3-3 test result, were predictive of disease duration $(3,6-10)$. However, these biomarkers are relatively difficult to measure in some areas due to cost and technical limitations. Therefore, it is necessary to find a biomarker that is easy to measure.

Measurement of red cell indices is cost-effective and routine, and these indices have been shown to be associated with disease progression in some neurodegenerative diseases such as Alzheimer's disease (AD). However, red cell indices have not been evaluated as biomarkers for CJD (11). The expression of $\mathrm{PrP}^{\mathrm{C}}$ on circulating red blood cells (RBCs) and the role of erythroid differentiation in CJD have been reported, which indicates that prions may play a role in hematopoiesis (12-14). The downregulation of erythroid genes and significantly decreased transcript levels of $\alpha$-hemoglobin stabilizing protein (AHSP) in prion-infected animals also demonstrated a connection between prion disease and peripheral erythropoiesis $(15,16)$. Based on previous studies, we hypothesized that peripheral blood erythrocyte indices may predict disease course in patients with CJD. Therefore, we investigated the association between red cell indices and survival time.

\section{METHODS}

\section{Ethics Statement}

This study was approved by the Ethics Committee of Xuanwu Hospital, Capital Medical University. All participants and/or their legal guardians provided written informed consent before undergoing any study procedures.

\section{Participants}

Participants were referred to the Department of Neurology, Xuanwu Hospital, Capital Medical University, from 2014 to April 2021. In total, 143 patients diagnosed with probable and definite (confirmed genetically) CJD based on standard diagnostic criteria were enrolled (17). Eight patients who had comorbid disorders that could potentially affect erythroid hematopoietic function, or other blood disorders, were excluded from this study. Telephonebased follow-ups were conducted by neurologists from our hospital to assess survival time. By the end of October 2021, 97 out of the 135 enrolled patients were deceased, 28 were living, and 10 were unable to be contacted (Figure 1). Survival time was defined as the time from onset of first symptom to death.

\section{Collection of Clinical Data}

Clinical data were collected for all enrolled patients. Electroencephalography (EEG), magnetic resonance imaging (MRI), and lumbar puncture were performed during hospitalization. All participants were administered the Barthel Index, a measure of functional severity commonly used to evaluate prion diseases $(18,19)$. MRI scans were performed on a 3.0 Tesla MRI system (Siemens Magnetom Trio Tim MRI system, Germany) using standard coil. T1-weighted, T2-weighted, fluidattenuated inversion recovery (FLAIR), diffusion-weighted image (DWI), apparent diffusion coefficient (ADC) data were acquired. EEG monitoring was performed using a 32-channel digital EEG system (DAVINCI-SAM, Micromed, Mogliano Veneto, Italy). Cerebrospinal fluid (CSF) and blood samples

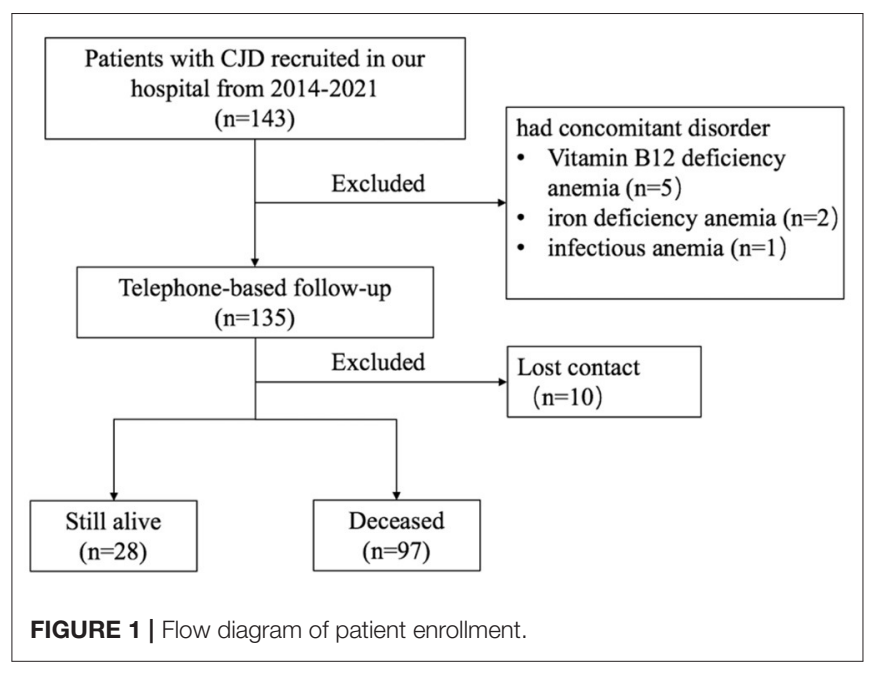

were collected by the medical staff in our hospital and transferred to the Chinese Center for Disease Control and Prevention, where CSF 14-3-3 protein was detected by western blot and the prion protein gene $(P R N P)$ was analyzed (20). Typical MRI imaging for CJD diagnosis was defined as the high signal of DWI or FLAIR in caudate/putamen or at least two cortical regions (temporal, parietal, occipital). Typical EEG pattern was defined as periodic sharp wave complexes (PSWCs) (17).

\section{Blood Sampling and Measurement of Blood Indices}

Blood samples from participants were collected between 6:00 a.m. and 7:00 a.m. and sent to the hospital lab for testing within one hour. Red blood cell indices included red blood cell (RBC) count, hemoglobin (Hb), hematocrit (HCT), mean corpuscular volume $(\mathrm{MCV})$, mean corpuscular hemoglobin $(\mathrm{MCH})$, mean corpuscular hemoglobin concentration $(\mathrm{MCHC})$, and red cell distribution width (RDW). In the case of blood samples collected at multiple time points after admission to the hospital, we used the first sample collected.

\section{Western Blot for 14-3-3 Protein in CSF}

A total of $20 \mu \mathrm{l}$ CSF sample was separated by $12 \%$ SDS-PAGE and electronically transformed onto nitrocellulose membrane (Whatman, USA). Blots were incubated in 1:1000 diluted 14-3-3 polyclonal antibodies (Santa Cruz, USA) and further incubated in 1:5000 diluted HRP-conjugated goat anti-Rabbit IgG (PerkinElmer, Germany). Reactive signals were visualized using an enhanced chemiluminescence kit (Amersham-Pharmacia Biotech, USA). Each test contained a preparation of goat brain tissue homogenate as the positive control. The 14-3-3 test was considered positive only when the 14-3-3 immunoreactivity was comparable with that of the positive control (21).

\section{Statistical Analysis}

Continuous variables are presented as the mean \pm standard deviation (SD) or median and interquartile range (IQR). Categorical data are presented as the frequency (percentage). 
TABLE 1 | Participant demographic and clinical characteristics.

\begin{tabular}{lc}
\hline Characteristic & No. (\%) \\
\hline Total, No. & 125 \\
Age at study visit, years & \\
$\quad$ Mean (SD) & $60.3(9.5)$ \\
$\quad$ Median (IQR) & $61.0(56.0-65.5)$ \\
$\quad$ Range & $30.0-82.0$ \\
Male/Female & $70 / 55$ \\
Creutzfeldt-Jakob disease & \\
Genetically confirmed & $5(4.0 \%)$ \\
$\quad$ Probable & $120(96.0 \%)$ \\
Barthel Index score at first visit & \\
Mean (SD) & $51.8(28.3)$ \\
Median (IQR) & $60.0(30.0-75.0)$ \\
Range & $0-95.0$ \\
A typical EEG, positive/total & $50(41.7 \%) / 120$ \\
CSF 14-3-3 (+) & $48(44.0 \%) / 109$ \\
A typical MRI brain scan, positive/total & $112(90.3 \%) / 124$ \\
Deceased & $97(77.6 \%)$ \\
Average time from first symptom to death, months & \\
Mean (SD) & $14.3(10.7)$ \\
Median (IQR) & $12.0(5-21.5)$ \\
Range & $2.0-51.0$ \\
$<=6$ months & $34(35.1 \%)$ \\
$<=12$ months & $54(55.7 \%)$ \\
$<=24$ months & $77(79.4 \%)$ \\
\hline
\end{tabular}

Spearman's rho correlation analysis was used to determine the associations between biochemical variables and survival time, and biochemical variables and Barthel Index. Mann-Whitney U test was performed to compare the erythrocyte indices between 14-3-3-positive patients and 14-3-3-negative patients. All red cell indices were binned in the median. Cox proportional hazard models were used to predict survival time. Three covariates (sex, age, Barthel Index) were fitted in three separate Cox proportional hazard models. All red cell indices were fitted in separate Cox proportional hazard models with and without covariates. A twosided $p$ value $<0.05$ was considered statistically significance. All statistical analyses were performed using SPSS, version 25 .

\section{RESULTS}

\section{Baseline Characteristics of the Sample}

A total of 125 patients with CJD were enrolled in this study, of whom 70 (56.0\%) were male. The mean age at diagnosis (SD) was 60.3 (9.5) years. Five of 125 patients were diagnosed with definite CJD (confirmed genetically), while 120 patients were diagnosed with probable CJD. Ninety-seven (77.6\%) patients were deceased at the time of data collection. Detailed demographic and clinical characteristics are shown in Table $\mathbf{1 .}$

Hemoglobin $(r=-0.263, p=0.009)$ and HCT $(r=-0.248$, $p=0.014)$ were significantly associated with survival time (Figure 2), and no red cell indices correlated with functional impairment level (measured by Barthel Index). Descriptive statistics for red cell index values are presented in Table 2. In addition, we analyzed the relationship between erythrocyte indices and 14-3-3 protein. No difference between 14-3-3positive group and 14-3-3-negative group (data not shown) was found.

\section{Variables Associated With Survival}

We first evaluated the associations of age, sex, and Barthel Index with survival. Age (HR 1.513, 95\% CI 1.006-2.276, $p=0.047$ ) and Barthel Index (HR 0.545, 0.354-0.839, $p=0.006$ ), but not sex, were significantly associated with survival time (Table 3 ). Younger age and higher baseline levels of function predicted longer disease duration. Interestingly, when both Barthel Index and age were entered simultaneously as predictors of survival, only Barthel Index remained statistically significant (HR 0.575, 95\% CI 0.371-0.891, $p=0.013$ ).

Each erythrocyte index was assessed as a predictor of survival time (Table 3). Higher baseline $\mathrm{Hb}$ and HCT were associated with shorter survival time, with $\mathrm{HR}$ values of 1.710 (95\% CI 1.124-2.600, $p=0.012$ ) and 1.689 (95\% CI 1.112-2.565, $p=$ 0.014 ) respectively, and this association remained significant after controlling for age, sex, and Barthel Index (Hb, HR 1.959, 95\% CI $1.232-3.116, p=0.004$; HCT, HR 1.927, 95\%CI 1.234-3.010, $p$ $=0.004$ ) (Figure 3). In contrast, RBC, MCV, MCH, MCHC, and RDW did not significantly correlate with survival with or without adjustment for the confounding effects of covariates.

\section{DISCUSSION}

We investigated the association between red cell indices and survival times in 125 patients with CJD. The results showed that higher $\mathrm{Hb}$ and HCT levels were associated with shorter survival times in patients with CJD. This was the first study to demonstrate the relationship between peripheral blood erythrocytes and disease duration in patients with CJD, and suggests that $\mathrm{Hb}$ and HCT may be potential prognostic indicators.

The median survival time in our study was 12 months (range: 2.0-51.0 months) and $55.7 \%$ of the patients in our study died within one year of onset of the first symptom. Although disease progression was slower in our study than that in other studies $(22,23)$, a survey performed by the Japanese CJD surveillance program showed a longer survival time, with a mean disease course of 17.4 months and $46.0 \%$ mortality within one year (24). Our study indicated that younger age was associated with longer disease duration, which was consistent with the results of previous studies $(3,22,25)$. As CJD is irreversible and fatal, the severity of the disease at baseline may predict prognosis. We found that lower baseline levels of function, as measured by Barthel Index, predicted shorter survival time (26).

In our study, $\mathrm{Hb}$ and $\mathrm{HCT}$ correlated with survival time in patients with CJD. Previous studies have found that both lower and higher hemoglobin levels were associated with an increased risk for developing $\mathrm{AD}$, and worse cognitive performance and faster cognitive decline, which suggests that erythrocytic hemopoiesis may be important in neurodegenerative processes 
A

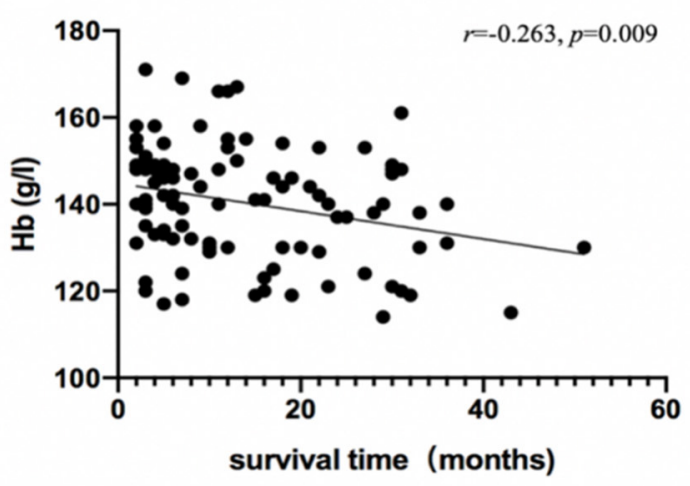

B

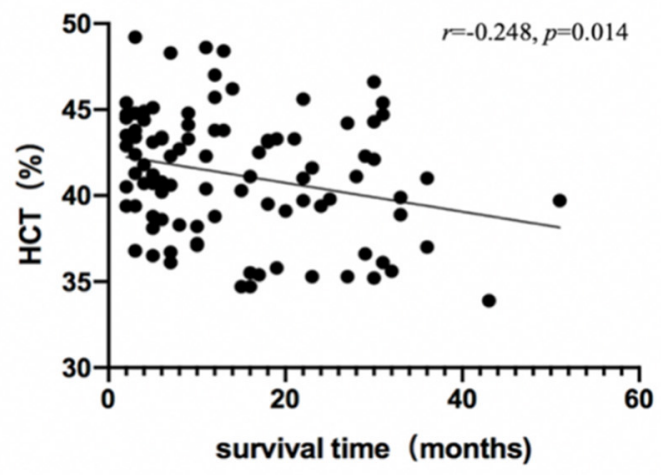

FIGURE 2 | Association between survival time and red cell indices. Hb (A) and HCT (B) levels in patients with CJD are plotted against survival time, and were analyzed using Spearman's rho correlation analysis.

TABLE 2 | Red cell indices and cohort characteristics of deceased patients.

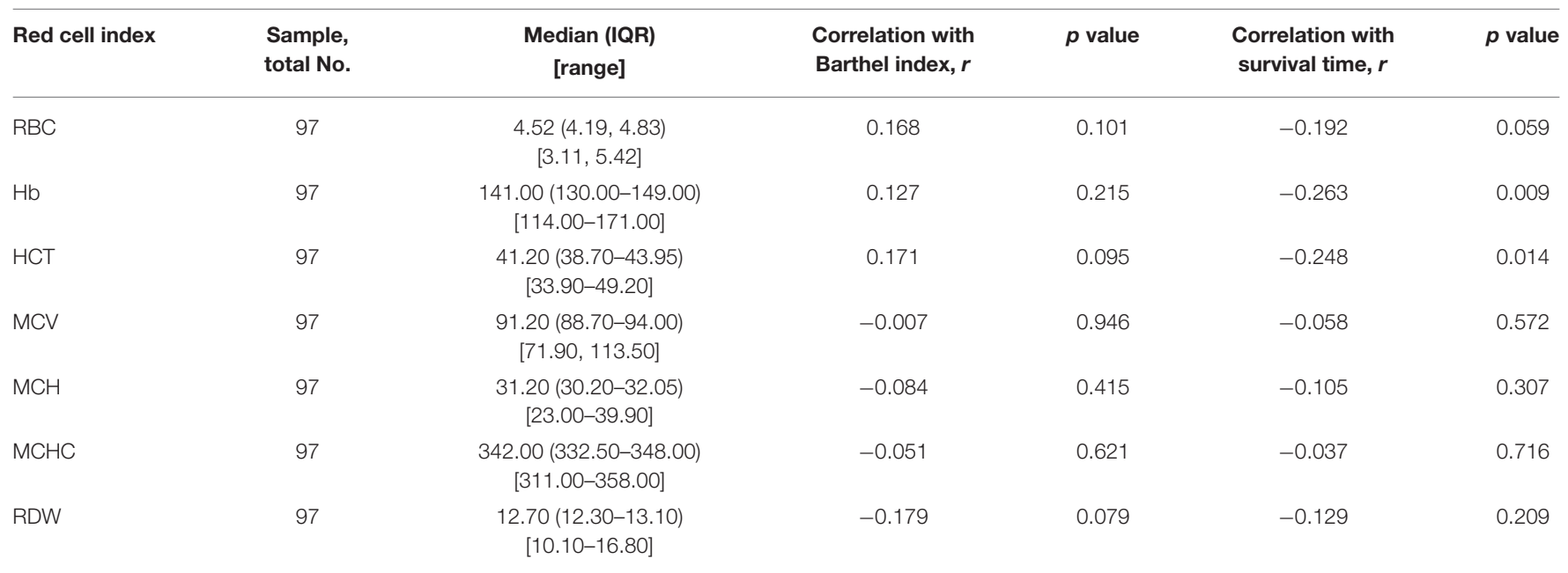

TABLE 3 | Cox proportional hazard models of the associations between red cell indices and survival.

\begin{tabular}{|c|c|c|c|}
\hline \multicolumn{3}{|c|}{ No Covariates } & Covariates \\
\hline HR & $95 \% \mathrm{Cl}$ & $p$ Value & HR \\
\hline
\end{tabular}

\section{Covariate}

\section{Age}

Sex

Barthel Index

Red cell Index

$\mathrm{RBC}$

$\mathrm{Hb}$

HCT

MCV

$\mathrm{MCH}$

$\mathrm{MCHC}$

RDW

$\begin{array}{ll}1.513 & 1.006-2.276 \\ 1.115 & 0.739-1.682 \\ 0.545 & 0.354-0.839 \\ & \\ 1.240 & 0.827-1.860 \\ 1.710 & 1.124-2.600 \\ 1.689 & 1.112-2.565 \\ 0.857 & 0.572-1.284 \\ 1.228 & 0.822-1.836 \\ 1.460 & 0.972-2.193 \\ 0.999 & 0.664-1.502\end{array}$

0.047
0.604
0.006
0.298
0.012
0.014
0.454
0.316
0.068
0.995

NA

NA

NA

1.374

1.959

1.927

0.788

1.133

1.504

0.954

$$
\begin{aligned}
& \text { NA } \\
& \text { NA } \\
& \text { NA }
\end{aligned}
$$




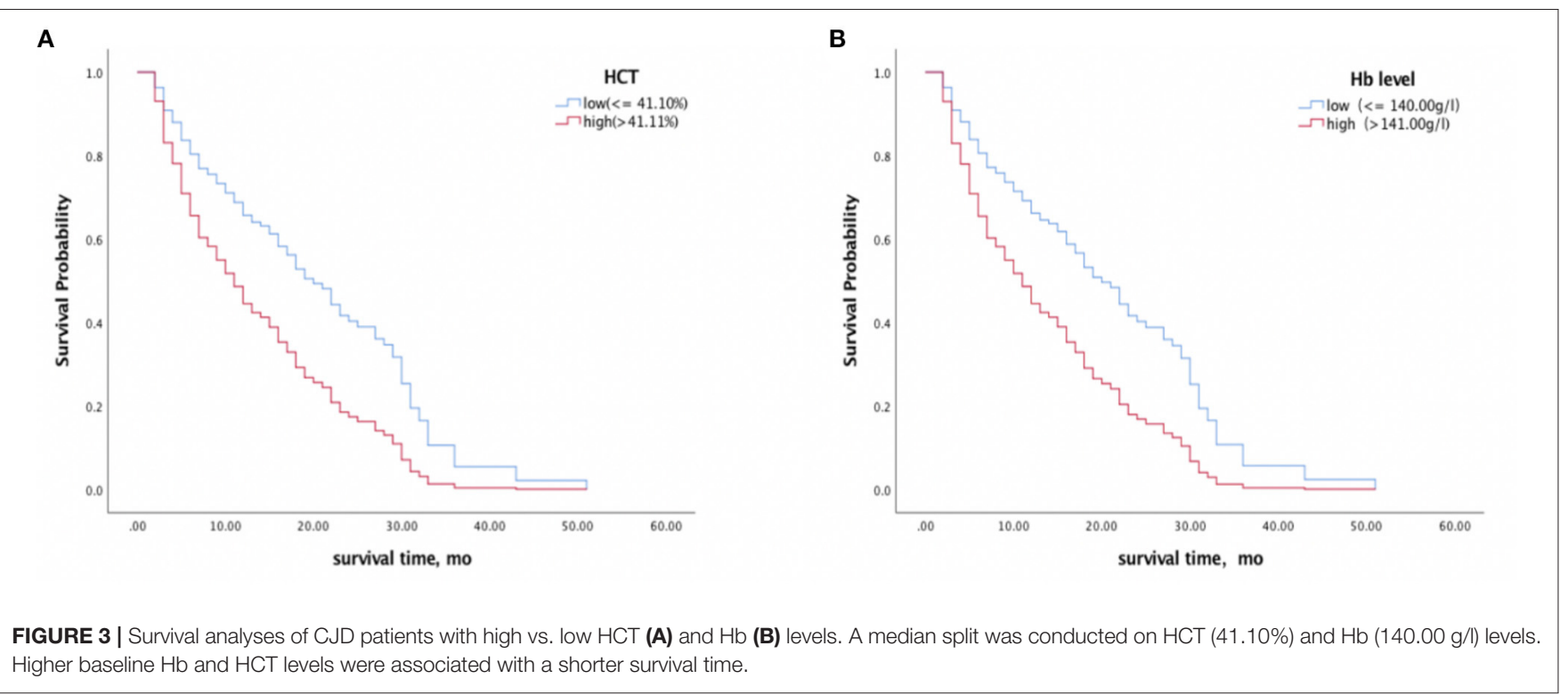

$(11,27)$. Some studies have focused on the effects of $\operatorname{PrP}^{\mathrm{C}}$ on homeostatic processes. Miele et al. (16) found a connection between prion pathogenesis and peripheral erythropoiesis through downregulation of the AHSP. Subsequent studies found that HBA2 was downregulated in cattle infected with atypical Bovine Spongiform Encephalopathy infected cattle, and reduced expression of several other erythroid genes, Kell, GPA, band 3, and ankyrin were observed in mice with prion disease $(15,28)$. These studies suggested that there was an association between erythrocytes and prion disease. The mechanisms linking $\mathrm{Hb}$ levels to disease duration in CJD patients have not been well-characterized, but upregulation of $\mathrm{Hb}$ has been discovered in $\mathrm{SCJD}$ brains, suggesting that it might be a compensatory mechanism of survival neurons (29). We hypothesized that downregulation of erythroid genes in patients with CJD may result in reduced $\mathrm{Hb}$ synthesis, but compensatory mechanisms may result in increased $\mathrm{Hb}$ synthesis in peripheral blood. Therefore, high levels of $\mathrm{Hb}$ may reflect the severity of prion pathology. In addition, high $\mathrm{Hb}$ and HCT levels may aggravate the disease via ischemic and hypoxic mechanisms, as reported in studies of $\mathrm{AD}$ and chronic obstructive pulmonary disease (associated with high $\mathrm{Hb}$ levels) $(11,30)$. Although mechanisms are unclear, it is possible that $\mathrm{Hb}$ and $\mathrm{HCT}$ could be associated with survival time in CJD. Furthermore, our results suggested that erythrocyte might be a potential therapeutic target. However, further studies are needed to verify our research results and to explore the mechanisms by which hematopoiesis and prion disease interact.

The Barthel Index from the date of sampling did not correlate with red cell indices, which indicated that red cell indices did not reflect disease severity in patients with CJD. This may also explain poor accuracy of the Barthel Index. Other assessment measures, such as the Medical Research Council (MRC) Prion Disease Rating Scale, could be used in further studies. 14-3-3 protein in CSF is considered to be a reliable biomarker for the diagnosis of CJD and relates to extensive brain tissue damage (31). Our result that the positive rate of 14-3-3 test was similar to those researches based on the Chinese population, but was much lower than those raised in other countries (32-35). We explain such a difference might be related to ethnic differences or most of the patients we recruit in the early disease stage. In addition, no significant correlation was found between 143-3 protein and erythrocyte indices, indicating that erythrocyte indices could not reflect acute neuronal destruction. Considering the limitation of the western blot technique in 14-3-3 evaluation, quantitative measurement by ELISA might be recommended in further study.

Our study had some limitations. The majority of patients with CJD recruited for our study were probable diagnoses without a pathological diagnosis. Although we were able to find an association between $\mathrm{Hb}, \mathrm{HCT}$, and survival time in CJD, we were unable to determine the underlying mechanisms. In the present study, we did not analyze PRNP codon 129 genotypes, which have been found to correlate with survival time. However, MM type accounted for $97.0-100.0 \%$ of all sCJD patients, according to previous studies performed in China (36-38). Therefore, the different patterns of codon 129 genotypes could not explain the results. Also, due to the multiple risk factors that may be associated with $\mathrm{Hb}, \mathrm{HCT}$, and survival time in CJD, we were not able to adjust for other factors that may be associated with these items.

\section{CONCLUSIONS}

This was the first study to investigate the relationship between red cell indices and survival time in patients with CJD patients, and our findings suggested that high $\mathrm{Hb}$ and HCT levels were associated with short disease duration. As red cell indices are frequently measured in current clinical practice, these results may 
have important value for estimating survival time in patients with CJD. However, the results should be interpreted with caution, and further studies are needed to confirm the results and explore the underlying mechanisms.

\section{DATA AVAILABILITY STATEMENT}

The original contributions presented in the study are included in the article/supplementary material, further inquiries can be directed to the corresponding author/s.

\section{ETHICS STATEMENT}

The studies involving human participants were reviewed and approved by the Ethics Committee of Xuanwu Hospital, Capital Medical University. The patients/participants

\section{REFERENCES}

1. Takada LT, Geschwind MD. Prion diseases. Semin Neurol. (2013) 33:34856. doi: 10.1055/s-0033-1359314

2. Johnson DY, Dunkelberger DL, Henry M, Haman A, Greicius MD, Wong K, et al. Sporadic Jakob-Creutzfeldt disease presenting as primary progressive aphasia. JAMA Neurol. (2013) 70:254-7. doi: 10.1001/2013.jamaneurol.139

3. Pocchiari M, Puopolo M, Croes EA, Budka H, Gelpi E, Collins S, et al. Predictors of survival in sporadic Creutzfeldt-Jakob disease and other human transmissible spongiform encephalopathies. Brain. (2004) 127(Pt 10):234859. doi: 10.1093/brain/awh249

4. Puoti G, Bizzi A, Forloni G, Safar JG, Tagliavini F, Gambetti P. Sporadic human prion diseases: molecular insights and diagnosis. Lancet Neurol. (2012) 11:618-28. doi: 10.1016/S1474-4422(12)70063-7

5. Llorens F, Rübsamen N, Hermann P, Schmitz M, Villar-Piqué A, Goebel S, et al. A prognostic model for overall survival in sporadic Creutzfeldt-Jakob disease. Alzheimers Dement. (2020) 16:1438-47. doi: 10.1002/alz.12133

6. Mead S, Burnell M, Lowe J, Thompson A, Lukic A, Porter MC, et al. Clinical trial simulations based on genetic stratification and the natural history of a functional outcome measure in Creutzfeldt-Jakob disease. JAMA Neurol. (2016) 73:447-55. doi: 10.1001/jamaneurol.2015.4885

7. Karch A, Hermann P, Ponto C, Schmitz M, Arora A, Zafar S, et al. Cerebrospinal fluid tau levels are a marker for molecular subtype in sporadic Creutzfeldt-Jakob disease. Neurobiol Aging. (2015) 36:19648. doi: 10.1016/j.neurobiolaging.2015.01.021

8. Skillbäck T, Rosén C, Asztely F, Mattsson N, Blennow K, Zetterberg H. Diagnostic performance of cerebrospinal fluid total tau and phosphorylated tau in Creutzfeldt-Jakob disease: results from the Swedish Mortality Registry. JAMA Neurol. (2014) 71:476-83. doi: 10.1001/jamaneurol.2013.6455

9. Zerr I, Schmitz M, Karch A, Villar-Piqué A, Kanata E, Golanska $\mathrm{E}$, et al. Cerebrospinal fluid neurofilament light levels in neurodegenerative dementia: Evaluation of diagnostic accuracy in the differential diagnosis of prion diseases. Alzheimers Dement. (2018) 14:751-63. doi: 10.1016/j.jalz.2017.12.008

10. Foutz A, Appleby BS, Hamlin C, Liu X, Yang S, Cohen Y, et al. Diagnostic and prognostic value of human prion detection in cerebrospinal fluid. Ann Neurol. (2017) 81:79-92. doi: 10.1002/ana.24833

11. Shah RC, Buchman AS, Wilson RS, Leurgans SE, Bennett DA. Hemoglobin level in older persons and incident Alzheimer disease: prospective cohort analysis. Neurology. (2011) 77:219-26. doi: 10.1212/WNL.0b013e318225aaa9

12. Holada K, Simak J, Brown P, Vostal JG. Divergent expression of cellular prion protein on blood cells of human and nonhuman primates. Transfusion. (2007) 47:2223-32. doi: 10.1111/j.1537-2995.2007.01451.x

13. Griffiths RE, Heesom KJ, Anstee DJ. Normal prion protein trafficking in cultured human erythroblasts. provided their written informed consent to participate in this study.

\section{AUTHOR CONTRIBUTIONS}

YK: original draft, data curation, and data analysis. ZC and JZ: review and editing. LW: supervision. All authors contributed to the article and approved the submitted version.

\section{FUNDING}

This work was supported by the Ministry of Science and Technology of China (Grant No. 2019YFC0118600), the National Natural Science Foundation of China (Grant No. 81971011), and the Beijing Municipal Science and Technology Committee (Grant Nos. D171100008217005 and 7202060).
Blood. (2007) 110:4518-25. doi: 10.1182/blood-2007-04-0 85183

14. Panigaj M, Glier H, Wildova M, Holada K. Expression of prion protein in mouse erythroid progenitors and differentiating murine erythroleukemia cells. PLoS ONE. (2011) 6:e24599. doi: 10.1371/journal.pone.0024599

15. Xerxa E, Barbisin E, Chieppa MN, Krmac H, Vallino Costassa E, Vatta P, et al. Whole blood gene expression profiling in preclinical and clinical cattle infected with atypical bovine spongiform encephalopathy. PLoS ONE. (2016) 11:e0153425. doi: 10.1371/journal.pone.0153425

16. Miele G, Manson J, Clinton M. A novel erythroid-specific marker of transmissible spongiform encephalopathies. Nat Med. (2001) 7:3614. doi: $10.1038 / 85515$

17. Zerr I, Kallenberg K, Summers DM, Romero C, Taratuto A, Heinemann U, et al. Updated clinical diagnostic criteria for sporadic Creutzfeldt-Jakob disease. Brain. (2009) 132(Pt 10):2659-68. doi: 10.1093/brain/awp191

18. Geschwind MD, Kuo AL, Wong KS, Haman A, Devereux G, Raudabaugh BJ, et al. Quinacrine treatment trial for sporadic Creutzfeldt-Jakob disease. Neurology. (2013) 81:2015-23. doi: 10.1212/WNL.0b013e3182a9f3b4

19. Haïk S, Marcon G, Mallet A, Tettamanti M, Welaratne A, Giaccone G, et al. Doxycycline in Creutzfeldt-Jakob disease: a phase 2, randomised, double-blind, placebo-controlled trial. Lancet Neurol. (2014) 13:1508. doi: 10.1016/S1474-4422(13)70307-7

20. Shi Q, Zhou W, Chen C, Zhang BY, Xiao K, Wang Y, et al. Rare E196A mutation in PRNP gene of 3 Chinese patients with Creutzfeldt-Jacob disease. Prion. (2016) 10:331-7. doi: 10.1080/19336896.2016.1190897

21. Shi Q, Chen C, Xiao K, Zhou W, Gao LP, Chen DD, et al. Genetic prion disease: insight from the features and experience of china national surveillance for Creutzfeldt-Jakob disease. Neurosci Bull. (2021) 37:157082. doi: 10.1007/s12264-021-00764-y

22. Chen C, Wang JC, Shi Q, Zhou W, Zhang XM, Zhang J, et al. Analyses of the survival time and the influencing factors of chinese patients with prion diseases based on the surveillance data from 2008-2011. PLoS ONE. (2013) 8:e62553. doi: 10.1371/journal.pone.0062553

23. Chen C, Dong XP. Epidemiological characteristics of human prion diseases. Infect Dis Poverty. (2016) 5:47. doi: 10.1186/s40249-0160143-8

24. Nagoshi K, Sadakane A, Nakamura Y, Yamada M, Mizusawa H. Duration of prion disease is longer in Japan than in other countries. J Epidemiol. (2011) 21:255-62. doi: 10.2188/jea.JE20100085

25. Collins SJ, Sanchez-Juan P, Masters CL, Klug GM, van Duijn C, Poleggi A, et al. Determinants of diagnostic investigation sensitivities across the clinical spectrum of sporadic Creutzfeldt-Jakob disease. Brain. (2006) 129(Pt 9):2278-87. doi: 10.1093/brain/awl159

26. Staffaroni AM, Kramer AO, Casey M, Kang H, Rojas JC, Orrú CD, et al Association of blood and cerebrospinal fluid tau level and other biomarkers 
with survival time in sporadic Creutzfeldt-Jakob disease. JAMA Neurol. (2019) 76:969-77. doi: 10.1001/jamaneurol.2019.1071

27. Shah RC, Wilson RS, Tang Y, Dong X, Murray A, Bennett DA. Relation of hemoglobin to level of cognitive function in older persons. Neuroepidemiology. (2009) 32:40-6. doi: 10.1159/000170905

28. Brown AR, Blanco AR, Miele G, Hawkins SA, Hopkins J, Fazakerley JK, et al. Differential expression of erythroid genes in prion disease. Biochem Biophys Res Commun. (2007) 364:366-71. doi: 10.1016/j.bbrc.2007.10.015

29. Tripathi AK, Singh N. Prion protein-hemin interaction upregulates hemoglobin synthesis: implications for cerebral hemorrhage and sporadic Creutzfeldt-Jakob disease. J Alzheimers Dis. (2016) 51:107-21. doi: 10.3233/JAD-151039

30. Ortapamuk H, Naldoken S. Brain perfusion abnormalities in chronic obstructive pulmonary disease: comparison with cognitive impairment. Ann Nucl Med. (2006) 20:99-106. doi: 10.1007/BF029 85621

31. Huang N, Marie SK, Livramento JA, Chammas R, Nitrini R. 14-3-3 protein in the CSF of patients with rapidly progressive dementia. Neurology. (2003) 61:354-7. doi: 10.1212/01.WNL.0000078890.89473.ED

32. Qi C, Zhang JT, Zhao W, Xing XW Yu SY. Sporadic Creutzfeldt-Jakob disease: a retrospective analysis of 104 cases. Eur Neurol. (2020) 83:6572. doi: $10.1159 / 000507189$

33. Chen S, He S, Xu YY, Teng HY, Zhang JW. Clinical presentation of sporadic Creutzfeldt-Jakob disease in Han-Chinese. Alzheimer Dis Assoc Disord. (2020) 34:188-90. doi: 10.1097/WAD.0000000000000350

34. Hermann P, Appleby B, Brandel JP, Caughey B, Collins S, Geschwind MD, et al. Biomarkers and diagnostic guidelines for sporadic Creutzfeldt-Jakob disease. Lancet Neurol. (2021) 20:235-46. doi: 10.1016/S1474-4422(20)30477-4

35. Chohan G, Pennington C, Mackenzie JM, Andrews M, Everington D, Will RG, et al. The role of cerebrospinal fluid 14-3-3 and other proteins in the diagnosis of sporadic Creutzfeldt-Jakob disease in the UK: a 10-year review. J Neurol Neurosurg Psychiatry. (2010) 81:1243-8. doi: 10.1136/jnnp.2009.197962

36. Gao C, Shi Q, Tian C, Chen C, Han J, Zhou W, et al. The epidemiological, clinical, and laboratory features of sporadic Creutzfeldt-Jakob disease patients in China: surveillance data from 2006 to 2010. PLoS ONE. (2011) 6:e24231. doi: 10.1371/journal.pone.0024231

37. Shi Q, Gao C, Zhou W, Zhang BY, Chen JM, Tian C, et al. Surveillance for Creutzfeldt-Jakob disease in China from 2006 to 2007. BMC Public Health. (2008) 8:360. doi: 10.1186/1471-2458-8-360

38. Qi C, Zhang JT, Zhao W, Xing XW Yu SY. Sporadic Creutzfeldt-Jakob disease: a retrospective analysis of 104 cases. Eur Neurol. (2020) 83:6572. doi: $10.1159 / 000507189$

Conflict of Interest: The authors declare that the research was conducted in the absence of any commercial or financial relationships that could be construed as a potential conflict of interest.

Publisher's Note: All claims expressed in this article are solely those of the authors and do not necessarily represent those of their affiliated organizations, or those of the publisher, the editors and the reviewers. Any product that may be evaluated in this article, or claim that may be made by its manufacturer, is not guaranteed or endorsed by the publisher.

Copyright (๑ 2022 Kong, Chen, Zhang and Wu. This is an open-access article distributed under the terms of the Creative Commons Attribution License (CC BY). The use, distribution or reproduction in other forums is permitted, provided the original author(s) and the copyright owner(s) are credited and that the original publication in this journal is cited, in accordance with accepted academic practice. No use, distribution or reproduction is permitted which does not comply with these terms. 